\title{
BOOK REVIEWS / RECENSIONI
}

\author{
Antonio Catàlfamo, Passato e presente, Bologna, Edizioni \\ Pendragon, 1993; L'eterno cammino, Bologna, Edizioni \\ Pendragon, 1995; Diario pavesiano, Bologna, Edizioni \\ Pendragon, 1999.
}

Anche se a distanza di tempo da una edizione all'altra dalla data di pubblicazione, le tre raccolte di poesie di Antonio Catààlfamo offrono, a mio parere, una comune chiave di lettura, un unico movimento interpretativo, in quanto in ogni puntata successiva viene proposta una rielaborazione di alcuni argomenti cari all'autore in cui si prefigge di prendere la misura del mondo che lo circonda. II primo, infatti, tratta la Sicilia delle sue origini, luogo di riflessione tra vita interna e tempo storico al quale va assoggettata la emotivitàà della memoria. Nel secondo, invece, si trova l'incontro con l'altro, il diverso, spesso in funzione di un viaggio come quello verso il Piemonte di Cesare Pavese, dal quale attingere, in senso spirituale, la dimensione del mito per far fronte alle intemperie della modernità: dal materialismo all'omologazione culturale; oppure, come l'approdo sull'isola di Cuba di Che Guevara per ritrovare, talvolta con uno sguardo bonariamente disincantato (in "Sogno cubano" si legge, per esempio, "Qui tutto è leggenda,/ anche se la zafra va male/ e i bambini muoiono di fame"), la freschezza di quegli ideali oramai distrutti nell'occidente dal capitalismo avanzato. Infine, quasi sempre in forma epigrammatica, c'è il dialogo con noti personaggi lontani e vicini, e con ciascuno, chiamato in presenza a seconda del caso, il poeta confida la propria perplessità davanti alle contraddizioni dell'esistenza.

Nato in provincia di Messina nel 1962 e malgrado la giovane età all'epoca delle raccolte sopra citate (alle quali bisogna aggiungere una primissima, Origini, scritta nel 1991), Antonio Catààlfamo è, a tutti gli effetti, uno scrittore impegnato, contando al suo attivo un libro di racconti e due volumi di saggi oltre a varie opere ancora inedite, e riscuotendo pertanto un ambito riconoscimento della critica nonché la 
vincita di vari premi letterari. "Ho letto", scrive Giorgio Bàrberi Squarotti nel suo elogio delle poesie, "con emozionata partecipazione. [...] La sua è una poesia spoglia, netta, martellata nell'incidere personaggi, situazioni, ricordi", e continua: "La sua poesia è di una rara perfezione nel raccogliere nel breve giro di ciascun testo tutto lo stupore un poco sorridente della vita che continuamente si rinnova".

A testimonianza del suo debito con Pavese, troviamo non solo le poesie, sparse qua e là nelle raccolte, dedicate allo scrittore langarolo e ai vari personaggi delle sue opere, ma anche la fida collaborazione presso il Centro di Studi Pavesiani nella cura di una collana di saggi internazionali di critica pavesiana. Catàlfamo condivide con la visione pavesiana del mondo l'incolmabile rottura che esiste tra mito personale, atemporale, rivolto alla natura, e i fatti della storia che con la loro violenza riflettono palesamente i giochi del potere, rottura, tra l'altro, che egli riporta a confronto della sua isola nativa e del tempo presente, come si nota dall'accostamento della "vigna" e "i biondi aranci" nella poesia "Sospensione" del Diario Pavesiano:

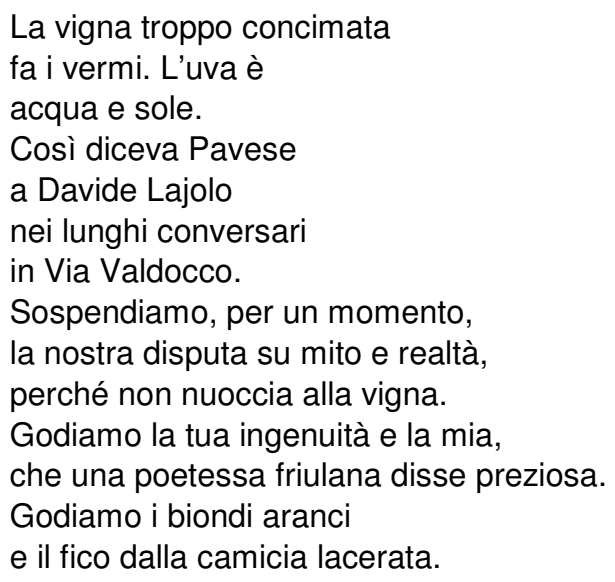

Sono forse le poesie dedite alla Sicilia, la terra delle sue radici, ad evocare un linguaggio davvero originale che, oscillando tra dialetto ed italiano, racchiude i colori e i sapori dell'isola e della sua gente. Le bellezze pittoriche del luogo, comunque, non servono solo a placare il 
dolore della nostalgia, ma si fanno segni di un mito interiorizzato, portatore, come le colline di Pavese, di una consapevolezza lacerante di veritàà inoppugnabile: l'ambivalenza inerente alla natura, tra forza generatrice e distruttiva, tra vita e morte, contro cui, si legge nella poesia "Cronache", è vano ogni sforzo umano:

Quest'anno l'estate

è in ritardo.

L'acqua di giugnu

ruvina lu mundu,

così dicono i vecchi contadini,

salutano e poi spariscono.

inghiottiti dalla salita polverosa.

Non serve aspettare

Marzo e le sue Idi.

Ogni stagione è buona per uccidere.

II sangue ingrassa la terra

e il cardo s'impiuma di viola.

La riflessione, frutto dell'intimo rapporto con il mito e della solitudine, è l'unico mezzo di conoscenza che rimane al poeta, oltre alla parola, per ridare un senso alla vita. A tal proposito, egli cerca un correlativo ai suoi presagi presso figure illustri, facendo loro appello in un gesto di fratellanza, con la speranza di rompere l'inquietudine del sapere. A volte, l'invito al colloquio acquista il tono amaro-ironico del rimprovero verso colui che ha scelto di vivere questa realtà diversamente dalla sua, come nel caso del conterraneo Leonardo Sciascia, in "Opzioni":

Il vento scuoteva i cedri, levigava i sassi morti

e le tue mani tremavano, afflitte dall'eterno tumore di questa terra agonizzante.

C'insegnavi ad essere imperfetti, come il seme che nulla produce

e si perde tra le spine e i rovi,

ad amare la ragione, fallace, nebulosa, 
contro la perfezione della scimmia, che suona l'eterna litania

e mostra il palmo della mano.

"C'è una vena contestataria, nella poesia di Catàlfamo", nota Paolo Ruffilli nella prefazione al Diario pavesiano, che lo porta a mirare, caparbiamente e coraggiosamente, "all'homo absconditus' che è dentro di noi; l'uomo anticonformista, solidale, altruista e inossidabile, che usa le parole che solo a lui appartengono, non la sintassi del potere". A mio avviso, èè questa la caretteristica della poesia di Catàlfamo che la rende più immediata e seducente.

Maria Grazia Sumeli Weinberg

(University of South Africa) 